\title{
Quantum diffusion of hydrogen
}

\author{
S DATTAGUPTA \\ School of Physical Sciences, Jawaharlal Nehru Unıversity, New Delhi 110067 , Indra
}

\begin{abstract}
tbstract. A brief overview is given on low temperature diffusive behaviour of $\mathrm{H}$ in $\mathrm{Nb}(\mathrm{OH})_{x}$, and measurement of diffusion, primarily by neutron scattering. We go in some detail into recent work on calculations of neutron structure factor as well as the diffusion coefficient of hydrogen, influenced by two-level excitations. Both the electronic and phononic contributions He covered. The importance of similar two-level excitations in insulating and metallic glasses is also pointed oul.
\end{abstract}

Keywords. Diffusion; hydrogen in metals; tunneling; neutron scattering; two-level systems: glasses.

\section{Introduction}

The problem of hydrogen in materials is one of the most interesting and important ones In modern solid state physics (Alefeld and Volkl 1978). Apart from several practical applications, some of which are discussed in this Proceedings, the problem has given rise to some challenging issues of quantum many body physics. Our objective in this paper is not to go into these issues in a very detailed way. We merely focus attention to the basic question of quantum diffusion of hydrogen. The hydrogen atom, being the lightest of the elements in nature, is believed to follow quantum laws as it moves from one lattice site to another, especially at low temperatures. The signature of this phenomenon can be found in numerous low-temperature measurements. We will however concentrate only on the experimental technique of neutron scattering (Springer and Richter 1987).

The reason that the dynamics of hydrogen in metals is considered intriguing can be assessed from a plot of the diffusion coefficient $D$ versus temperature (figure 1). If we compare the data for hydrogen with other interstitials such as nitrogen, oxygen or carbon we find that the $D$ for hydrogen is orders of magnitude larger (Hempelmann et al 1989). In fact, the value of $D$ around and above the room temperature is about $10^{-5} \mathrm{~cm}^{2} / \mathrm{sec}$, which is similar to that for liquid-state diffusion. The data for $D$ can be derived from 'quasielastic' neutron scattering which is described by a structure factor $\mathrm{S}(\mathbf{Q}, \omega)$ that can be often fitted to a Lorentzian lineshape (figure 2):

$$
S(\mathbf{Q}, \omega)=\frac{1}{\pi} \frac{\Gamma(\mathbf{Q})}{\pi \omega^{2}+\Gamma^{2}(\mathbf{Q})}
$$

Here $\mathbf{Q}$ is the wave-vector transfer and $\omega$ the frequency transfer in the scattering process. The 'width' $\Gamma(\mathbf{Q})$ carries information on $D$ because, in the small $Q$-domain it can be written as

$$
\Gamma(\mathbf{Q})=D Q^{2}
$$

Most of our discussion here will be restricted to hydrogen in niobium which has a bcc 


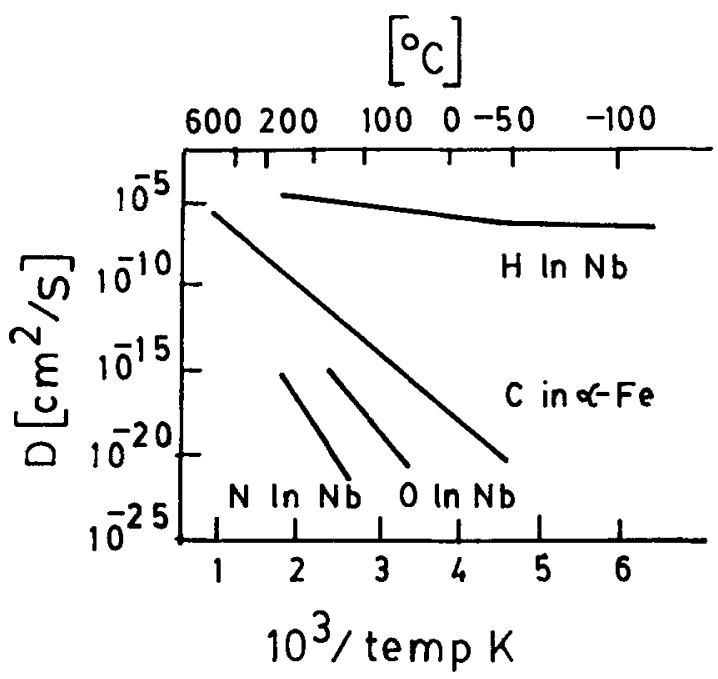

Figure 1. Comparative data on interstitial diffusion.

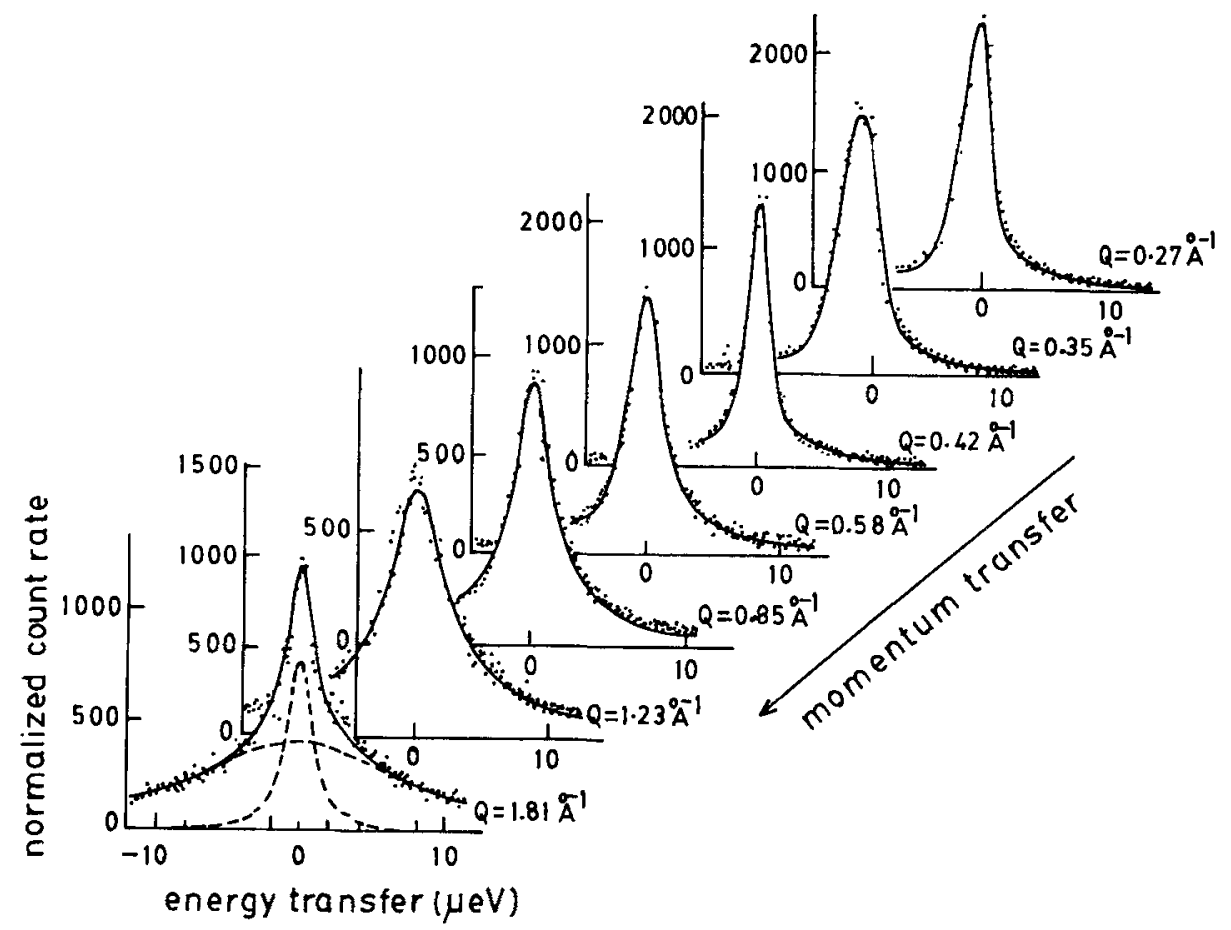

Figure 2. Typical quasielastic neutron spectra.

structure. Ordinarily, the hydrogen occupies twenty four interstitial sites per unit cell of niobium (figure 3). But, for the purpose of a focussed treatment of quantum diffusion, we shall further specialize to the case of niobium containing other interstitial impurities such as oxygen, nitrogen or carbon (figure 4). These interstitial defects are effectively 
immobile in the temperature regime in which hydrogen moves about rapidly (see figure 1), but they can act as trapping centres for hydrogen (Morkel et al 1978). Thus, consider figure 4 in which, in a sample of $\mathrm{Nb}(\mathrm{OH})_{x}$, the oxygen occupies an octahedral interstitial site, indicated by a solid circle, while the hydrogen can occupy any of the tetrahedral sites marked as a, b, c, d or e. It is borne out, however, by numerous earlier experiments as well as lattice statics calculations, that it is the pair of sites marked e (encircled) that the hydrogen preferentially occupies (Magerl et al 1983; Wipf and Neumaier 1984).

Our model for the dynamics of hydrogen in $\mathrm{Nb}(\mathrm{OH})_{x}$ in the temperature range of 0 and $160 \mathrm{~K}$ is therefore as follows. The host matrix atoms including the trapping defect are viewed to be rigidly fixed at their respective sites. In this background, the hydrogen moves in a double-well potential, the minima of which are separated by about $1.1 \mathrm{~A}$ (figure 5). This potential has an asymmetry, caused by long-range strain interactions between $\mathrm{OH}$-pairs. On the other hand, the concentration $x$ is so small that it suffices to consider only a pair of oxygen and hydrogen, at a time (Grabert and Wipf 1990). With the preceding remarks on the simplified model, which provide a setting for our rather restricted analysis of quantum diffusion of hydrogen, the plan of this paper may be

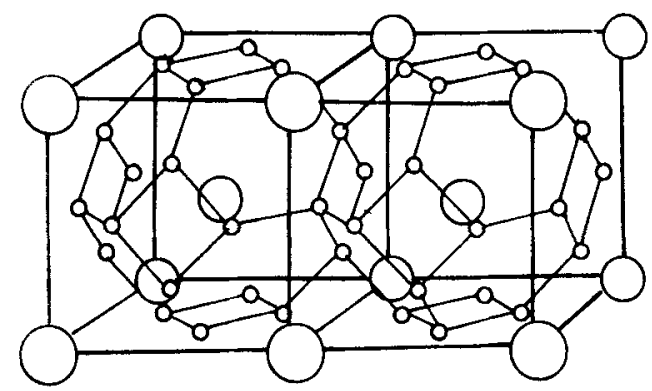

Figure 3. Lattice of bcc niobium with tetrahedral interstitial sites.

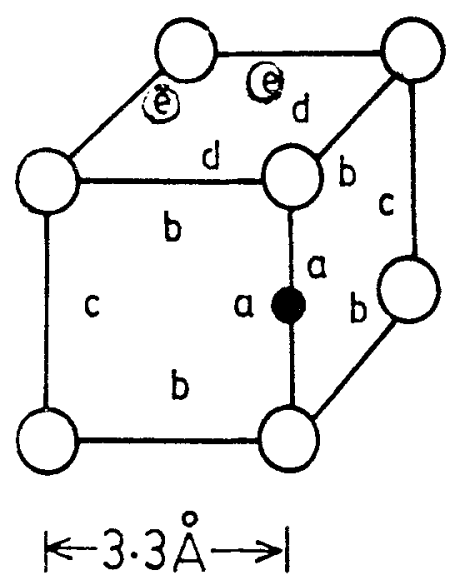

Figure 4. The bec niobium unit cell with an oxygen (solıd circle) on an octahedral interstitial site. The letters $a, b, c, d$ and $e$ indicate the positions of tetrahedral interstitial sites located on the visible surfaces of the unit cell. The trapped hydrogen is believed to occupy the sites labelled by the letter $\mathrm{e}$. 


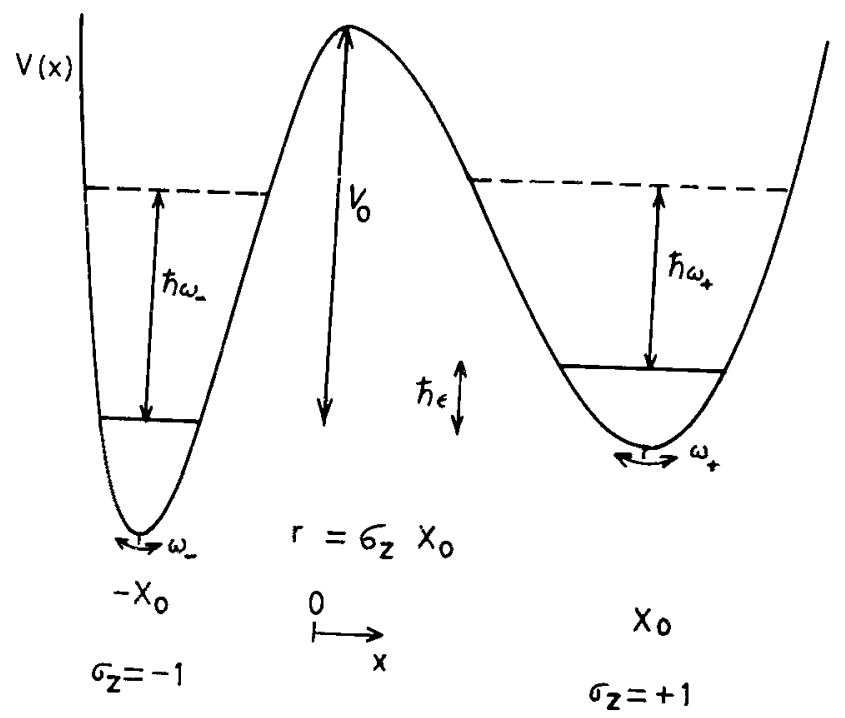

Figure 5. The double-well potential for $\mathrm{H}$ in $\mathrm{Nb}(\mathrm{OH})_{x}$, in the two-state limit.

stated as follows. In section 2, we present first the evidence of the tunneling motion of hydrogen in the ultra-low-temperature data on neutron scattering. We discuss next the data on the 'quasielastic' line, the width of which reveals the jump rate of the hydrogen from one minimum of the double well to another. Section 2 also contains a sketch of the theoretical formalism for the structure factor, which forms the starting point of our model calculation, summarised in section 3. Here, we argue that one and the same Hamiltonian, popularly known as the spin-boson Hamiltonian in the recent literature, is capable of handling both the electron-dominated low-temperature regime $(10 \mathrm{~K}<T<60 \mathrm{~K})$ as well as the phonon-dominated intermediate-temperature regime $(60 \mathrm{~K}<T<160 \mathrm{~K})$. In section 4 we put the problem of hydrogen in $\mathrm{Nb}(\mathrm{OH})_{x}$ in the more general context of two-level systems in glasses. We argue that much of our theoretical treatment, summarised in section 3, can be adapted to the case of lowtemperature dynamics of glasses. Section 5 contains a few concluding remarks. It is fair to emphasize here itself that most of the presented results are already available in published literature. This paper is merely an overview of the subject.

\section{Structure factor}

\subsection{Experimental data}

We show in figure 6 the low-temperature data on the neutron scattering measurements. Because niobium is a superconductor, experiments are done in an external magnetic field $\mathrm{H}$ of 0.7 tesla so that superconductivity is quenched, and one has to deal with the normal state electrons only. The plotted structure factor $\mathrm{S}(Q, \omega)$ versus the "energy transfer" $\omega$ exhibits characteristic tunneling peaks (Wipf et al 1987). These peaks show up as inelastic lines on either side of the central peak. The central peak has contribution from other hydrogen atoms in the sample in addition to the trapped hydrogen 


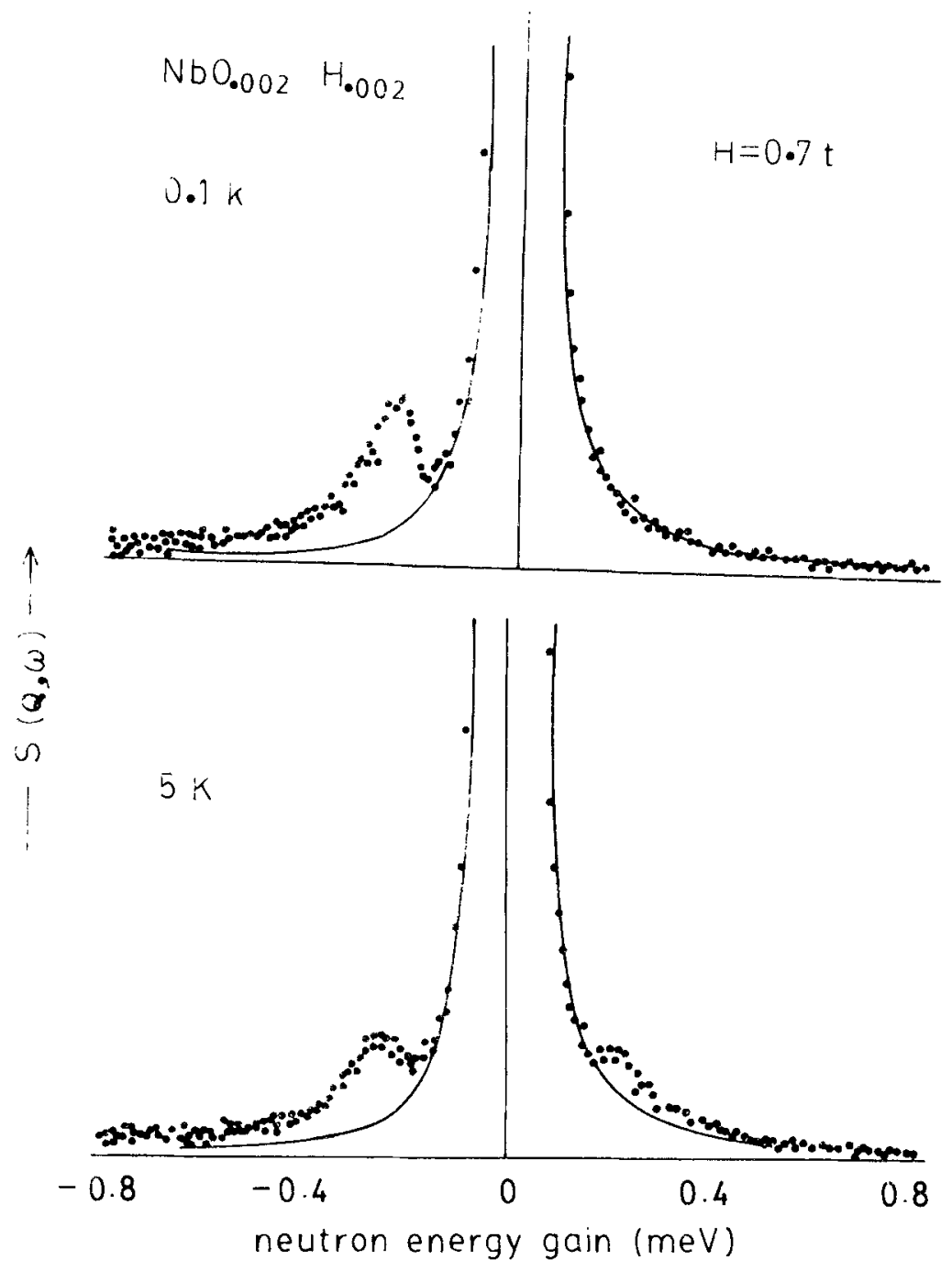

Figure o. Veutson spectra of a $\mathrm{Nb} \mathrm{O}_{0002} \mathrm{H}_{0002}$ sample at $0.1 \mathrm{~K}$ and $5 \mathrm{~K}$. taken in the wormal conducting electronic state. by subjecting the sample to a field of 0.7 tesla.

atoms. This other contribution, therefore, has to be subtracted from the background in order to extract the features which can be attributed to just the hydrogen-dynamics in a double-well potential. We notice that the pair of inelastic peaks is more pronounced at $5 \mathrm{~K}$ than at $0 \cdot 1 \mathrm{~K}$. This is purely due to the quantum-mechanical detailed balance factor. important at low temperatures. The same factor also determines the intensities of the Stokes and anti-Stokes lines in Raman spectroscopy. We may further remark that the inelastic peaks are associated with the 'tunneling mode' that arises from coherent clock-like motion of hydrogen from one minimum of the double-well to another.

As the temperature is raised, coherence is partially destroyed. due to both themal fluctuations as well as interactions with other degrees of freedom in the system. What 
these degrees of freedom are, will be clarified in the sequel, but at this stage it is sufficient to say that in this so-called incoherent tunneling regime the inelastic lines shift towards the centre and eventually merge into the central line (Steinbinder et al 1988). One obtains then a quasielastic line, the name 'elastic' originating from the fact that this line is centred on $\omega=0$, but it is still dominated by the dynamics of hydrogen (figure 7). The dynamics in this regime can be thought of as classical-like thermal hops from one bottom of the double-well to another. But, it is important to remember that quantum mechanics still influences the dynamical behaviour.

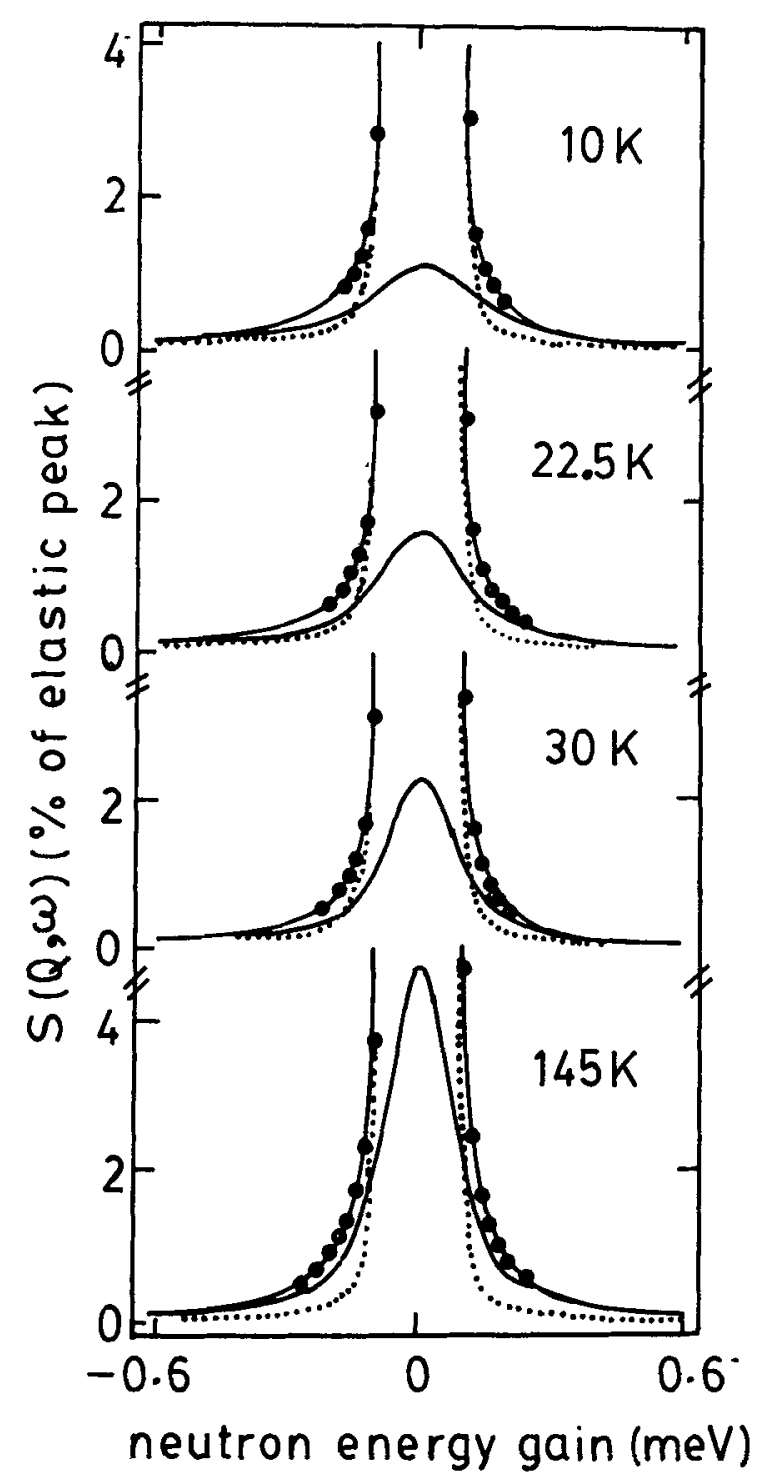

Figure 7. Quasielastic neutron spectra of $\mathrm{Nb} \mathrm{O}_{0.022} \mathrm{H}_{0.002}$ sample. The thin and the thick solid curves are fit results for the quasielastic and total scattering intensities. The broken curves indicate the resolution measured with a vanadium standard. 
As mentioned earlier, the temperature-dependence of the hopping rate can be extracted from the width of the quasielastic line. The data for the latter are shown in figure 8 (Steinbinder et al 1988). One of the most intriguing aspects of the lowtemperature diffusion of light interstitials such as hydrogen and (positive) muon is exhibited here; the jump rate $\Gamma$, instead of increasing with temperature, actually decreases (Hempelmann et al 1989). What is also significant is that the temperaturedependence can be fitted to a power-law:

$$
\Gamma \propto T^{2 K-1}
$$

We shall comment on the meaning of the parameter $K$ as well as the physical origin of this power-law dependence later in section 3 .

\subsection{Formalism}

It is well-known that the scattering of neutron from hydrogen in materials is predominantly 'incoherent' (Richter 1983; Dattagupta 1987). (This nomenclature should not be confused with the one employed earlier in the context of tunneling.) Incoherent scattering implies that one has to consider the involvement of the same hydrogen in the scattering process. Because the neutron undergoes a phase shift $\exp (-i \mathbf{Q} \cdot \mathbf{r})$, where $\mathbf{r}$ is the instantaneous position of the scatterer (i.e., hydrogen, in the present instance), it is the correlation of this quantity with itself at a later time that determines the observed structure factor. An expression for the latter can then be written as

$$
S(\mathbf{Q}, \omega)=\int_{-\jmath}^{\infty} \mathrm{d} t \exp (i \omega t)\langle\exp (-i \mathbf{Q} \cdot \mathbf{r}(0)) \exp (i \mathbf{Q} \cdot \mathbf{r}(t))\rangle,
$$

where the angular brackets represent thermal averaging. An important point to note

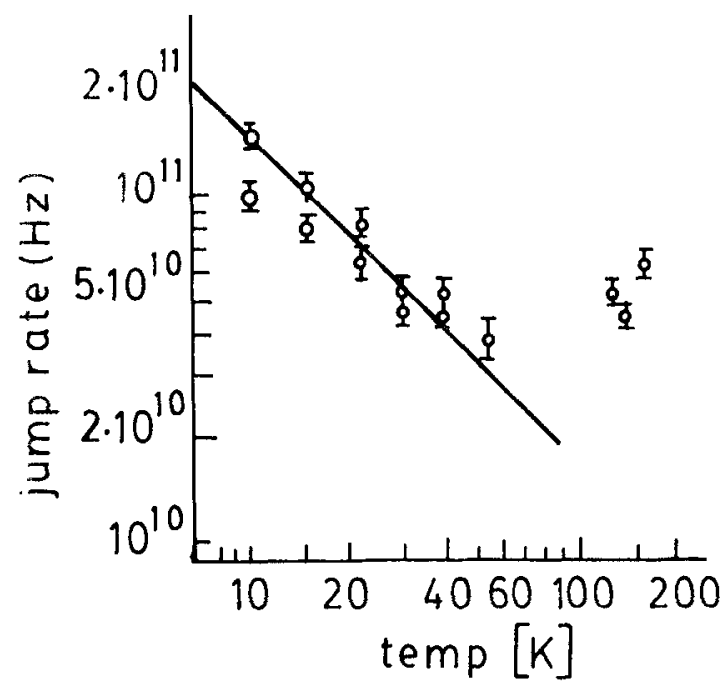

Figure 8. Hopping or jump rate of the trapped hydrogen in $\mathrm{Nb} \mathrm{O}_{0.002} \mathrm{H}_{0.002}$ samples. The fump rate has a temperature dependence proportional to $T^{2 K-1}$. 
here is that $\mathbf{r}$ is a quantum-mechanical operator which does not commute with itself at different times.

We refer again to the asymmetric double-well potential (figure 5) and highlight the different energy scales in the problem, some relevant, some not (Dattagupta et al 1989). The most relevant energy (or frequency) parameters are the tunneling energy $h \Delta_{0}\left(\Delta_{0}\right.$ is the corresponding tunneling frequency' calculable, say, in the WKB limit), the asymmetry energy $\hbar \delta$ and the thermal energy $K_{\mathrm{B}} T$ - they are all in the meV range, for $\mathrm{Nb}$ $(\mathrm{OH})_{x}$. These energy scales are well separated, by about three orders of magnitude, from the three other comparable parameters: the harmonic oscillator energy in either well $\hbar \omega_{0}$, the characteristic phonon energy $\hbar \omega_{\mathrm{D}}\left(\omega_{\mathrm{D}}\right.$ being the Debye frequency), and the Fermi energy $\varepsilon_{\mathrm{F}}$. This implies that the low-temperature dynamics of hydrogen is effectively restricted to a two-dimensional Hilbert space comprising the two lowest energy levels of the system. The hydrogen in $\mathrm{Nb}(\mathrm{OH})_{x}$, therefore, provides a unique example of what has come to be known in the literature as two-level systems (TLS).

In view of the TLS nature of excitations that are dominant at low temperatures, the position operator $\mathbf{r}$ can be mapped into a pseudo-spin operator $\sigma_{\mathrm{Z}}$ :

$$
\mathbf{r}=\hat{\imath} \sigma_{\mathrm{Z}} x_{0}
$$

Here we have assumed, without loss of generality, that the potential is a one-dimensional one along the unit vector $\hat{\imath}$ and $\sigma_{2}$ is a Pauli matrix which takes two values -1 or +1 , depending on whether the hydrogen is in the left or the right well, respectively. Because of the special property of $\sigma_{Z}\left(\right.$ viz. $\left.\sigma_{Z}^{2}=1\right)$, the operator $\exp (-i \mathbf{Q} \cdot \mathbf{r})$ can be simplified drastically, reducing the structure factor to:

$$
S(\mathbf{Q}, \omega)=f_{1}\left(Q \cdot x_{0}\right) \delta(\omega)+f_{2}\left(Q \cdot x_{0}\right)\left[1+\exp (\beta h(\omega)]^{-1} \operatorname{Re}\left(\lim _{\delta \rightarrow 0} \tilde{C}(p)\right) .\right.
$$

Here $f_{1}$ and $f_{2}$ are the so-called form factors which depend on the dimensionless quantity $Q \cdot x_{0}$ and $\tilde{C}(p)$ is the Laplace transform of the 'symmetrized' correlation function of $\sigma_{7}$,

$$
\tilde{C}(p)=\int_{0}^{\infty} \mathrm{d} t \exp (-p t) \frac{1}{2}\left[\left\langle\sigma_{Z}(0) \sigma_{Z}(t)\right\rangle+\left\langle\sigma_{Z}(t) \sigma_{Z}(0)\right\rangle\right], \quad p=-i \omega+\delta .
$$

The problem of the tunneling dynamics of the TLS and the evaluation of the structure factor then reduce to the calculation of the correlation function $\widetilde{C}(p)$ (Dattagupta et al 1989).

\section{The spin-boson Hamiltonian}

Before we set out to write down the form of the basic Hamiltonian for the problem at hand it is pertinent to discuss the physics that motivates it (Grabert and Schober 1994). As mentioned earlier, below about $5 \mathrm{~K}$, the hydrogen moves from one well to the other as an independent quantum particle decoupled from its surroundings. This decoupling occurs in the Born-Oppenheimer $(\mathrm{B}-\mathrm{O})$ sense, as the hydrogen dynamics is much faster than the dynamics of the host atoms (niobium as well as the 'trap' i.e. oxygen). Thus phonons are unimportant at very low temperatures because ordinarily, they move much slower than hydrogen. 
However, when the temperature exceeds $5 \mathrm{~K}$, the electron-hole excitations whose density of states has a low-frequency tail become thermally accessible. We have then a failure of the $\mathrm{B}-\mathrm{O}$ approximation as it were, and the otherwise 'adiabatic' coupling between the TLS and the conduction electrons gives way to a "nonadiabatic' part. It is this nonadiabaticity, characterising the drag on the hydrogen by the electron-hole pairs, that is believed to be the dominant effect in the incoherent tunneling regime $(10 \mathrm{~K}<T<60 \mathrm{~K})$. The result is a power-law dependence of the jump rate on the temperature, first pointed out by Kondo in the context of muon diffusion in metals. An important theoretical point to note here is that each electron-hole pair can be represented by a boson and hence an appropriate bosonized form of the environmental interaction yields a simplified analysis (see below).

As the temperature exceeds $60 \mathrm{~K}$ or so, short wavelength phonon states are populated. They now constitute the dominant density-of-states-factor, leading to a coupling between the TLS and phonons-- note again a breakdown of the $\mathrm{B}-\mathrm{O}$ approximation! The influence of phonons on the hydrogen can be treated as in the "small-polaron" theory (Holstein 1959).

With the preceding remarks on the basis of assuming the bosonized nature of the excitations of the environment, be it electron-hole pairs or phonons, the Hamiltonian can be written as

$$
\mathscr{H}=\hbar \Delta_{0} \sigma_{x}+\hbar \varepsilon \sigma_{\lambda}+\sigma_{7} \sum_{i} G_{j}\left(b_{j}+h_{j}^{\dagger}\right)+\sum_{j} \hbar \omega_{j} b_{j}^{\dagger} b_{j} .
$$

Equation (7) represents the so-called spin-boson Hamiltonian which has been used extensively in the recent literature in the context of the dissipative dynamics of a quantum two-level system (TLS) (Leggett et al 1987). The first two terms account for the internal dynamics of the TLS, whereas the third term denotes linear coupling between the TLS and the bosonic excitations of the environment, customarily called the 'heat-bath'. The bosonic excitations are written in terms of the second-quantized creation and annihilation operators $b_{i}^{+}$and $b_{j}$ while $G_{j}$ is a suitable coupling constant.

It is clear that the first two terms lead to coherent dynamics of the TLS. Incoherence arises from dissipative or frictional effects due to the coupling with the bosons, whose energy levels must form a continuum in order to simulate heat-bath effects. This motivates the introduction of a 'spectral density' $J(1)$ which characterizes the density of states of the heat-bath excitations:

$$
J(\omega) \equiv \frac{2}{h^{2}} \sum_{j} G_{j}^{2} \delta\left((1)-\omega_{j}\right) .
$$

The spectral density $J((1))$ occuples a central position in the theory, the form of which is governed by the nature of the elementary excitations. Thus, electron-hole pairs are represented by a linear dependence of $J(1)$ ) on $\omega$, whereas phonons are described by $J(\omega)$ being proportional to $\omega^{2 s+1}$, where the value of $s$ depends on the kind of phonons that are relevant. This point then underscores our earlier remark that the same Hamiltonian in (7) can account for both conduction electrons and phonons. Hence, a common form of the spectral density can be introduced as (Grabert and Schober 1994):

$$
J(\omega)=\left(K(1)+U \omega^{2 s+1}\right) \exp \left(-(\omega) /()_{c}\right)
$$


The coupling constant $K$ has been already introduced in relation to the temperaturedependence of the jump rate in the incoherent tunneling regime (see the last paragraph of $\S 2$ ); it parametrizes the influence on the TLS of conduction electrons. Similarly the coupling parameter $U$ accounts for the interaction between the TLS and the short wavelength phonons. The exponential term in (9) is a necessary theoretical construct, involving a high-frequency cut off $\omega_{\mathfrak{c}}$, which has no physical consequences but may be chosen to be of the order of either the Debye frequency $\omega_{\mathrm{D}}$ or the Fermi frequency $\omega_{\mathrm{F}}\left(=\varepsilon_{\mathrm{F}} / \hbar\right)(\mathrm{cf} . \S 2.2)$

We have shown earlier how an appropriate perturbation theory treatment of the coupling term (proportional to $G_{j}$ ) in the Hamiltonian in (7) leads to a complete analysis of the neutron scattering lineshape, including the occurrence of the inelastic peaks in the coherent tunneling regime and the collapse and merger of the inelastic peaks with the central line (Dattagupta and Qureshi 1991; Qureshi 1994). As our primary interest in the present paper is in studying quantum diffusion of hydrogen, we concentrate here only on the incoherent tunneling regime. The correlation function, in this regime, can be written in terms of a memory-kernel $\tilde{\phi}(p)$ :

$$
\tilde{C}(p)=(p+\tilde{\phi}(p))^{-1} .
$$

The jump rate $\Gamma$ can be extracted from the Markovian limit of $\tilde{\phi}(p)$ :

$$
\Gamma=\frac{1}{2} \widetilde{\phi}(p=0)
$$

We may now summarize the results for the jump rate $\Gamma$, which are written below for the symmetric case $(\varepsilon=0)$ only, as the expressions for the asymmetric situation $(\varepsilon \neq 0)$ are somewhat more complicated. The jump rate $\Gamma$ can be expressed in terms of a timedependent 'decay function' $\Lambda(t)$ (Grabert and Schober 1994):

$$
\Gamma=\frac{1}{4} \Delta_{0}^{2} \int_{-\infty}^{\infty} \mathrm{d} t \exp (-\Lambda(t))
$$

where

$$
\Lambda(t)=2 \int_{0}^{\infty} \mathrm{d} \omega \frac{J(\omega)}{\omega^{2}}\left[\cot h\left(\frac{1}{2} \hbar \beta \omega\right)-\frac{\cos (\omega t)}{\sin h\left(\frac{1}{2} \hbar \beta \omega\right)}\right] .
$$

The decay function, with the aid of (9), can then be split into the 'electronic' and 'phononic' parts:

$$
\Lambda(t)=\Lambda_{\mathrm{el}}(t)+\Lambda_{\mathrm{ph}}(t)
$$

which further lead to

and

$$
\Gamma_{\mathrm{el}}=\frac{\Gamma(K)}{\Gamma(1-K)} \frac{1}{2} \Delta_{0}\left(\frac{2 \pi K_{\mathrm{B}} T}{\hbar \Delta_{0}}\right)^{2 K-1},
$$

$$
\Gamma_{\mathrm{ph}}=\left(\frac{\pi}{16 E_{\mathrm{c}} K_{\mathrm{B}} T}\right)^{1 / 2} \Delta_{0}^{2} \exp \left(-\frac{E_{\mathrm{c}}}{K_{\mathrm{B}} T}\right) .
$$

In (15), $\Gamma(K)$ is a gamma function and the term proportional to $T^{2 K-1}$ is the power-law dependence, discussed earlier to be the consequence of nonadiabatic 
interaction between the TLS and the conduction electrons. Equation (16), on the other hand, yields the phononic contribution to the jump rate which, though of the Arrhenius form, has a very different origin, that is, quantum mechanical. In fact, it can also be calculated from the 'golden rule' of perturbation theory in which the tunneling term is treated up to second order (Flynn and Stoneham 1970; Kagan and Klinger 1974). The parameter $E_{\mathrm{c}}$ is the so-called 'coincidence energy', given in terms of the phononic part of the spectral density, as:

$$
E_{\mathrm{c}}=\frac{1}{2} \hbar \int_{0}^{\infty} \mathrm{d} \omega \frac{J_{\mathrm{ph}}(\omega)}{\omega}
$$

We may therefore use (10), in conjunction with (11), (15) and (16) to interpret the data on the jump rate $\Gamma$ (see figure 8 ) over a wide range of temperatures, from $10 \mathrm{~K}$ to $60 \mathrm{~K}$ in the electron-dominated region and from $60 \mathrm{~K}$ to $150 \mathrm{~K}$ or so, in the phonon-dominated domain.

\section{Two-level systems in glasses}

The quantum diffusion of hydrogen in $\mathrm{Nb}(\mathrm{OH})_{x}$ provides an archetypal example of the low-temperature dynamics of TLS in metals. Fortunately, as discussed in the paper, a lot of unambiguous experimental results are available on this system which can be subjected to detailed theoretical analysis. We have shown here that the analysis is based upon certain recent advances made in the theory of tunneling systems, which also bears a close resemblance to the theory of the Kondo effect (Qureshi and Dattagupta 1994). Hence, the hydrogen in $\mathrm{Nb}(\mathrm{OH})_{x}$ is a critical testing ground of these contemporary theoretical developments. It is therefore legitimate to ask whether the theory, developed so successfully for hydrogen in $\mathrm{Nb}(\mathrm{OH})_{x}$, can be also applied to other systems in which TLS excitations are important.

One such system of great significance in materials science is glass at low temperatures. It was discovered almost twentyfive years ago by Pohl and his collaborators that numerous insulating glasses exhibit universal thermodynamic properties (Narayanamurti and Pohl 1970; Zeller and Pohl 1971; Pohl et al 1974). This is exemplified in figure 9 where the specific heat $C(T)$, divided by the Debye contribution proportional to $T^{3}$, is plotted against the temperature $T$. The comparative data on crystalline $\alpha$-quartz and its amorphous version, vitreous silica show that while the Debye behaviour is well-satisfied for quartz up to about $5 \mathrm{~K}$, the specific heat for vitreous silica differs significantly on two counts. One, the magnitude of the lowtemperature specific heat for the glassy system is two orders of magnitude larger than its crystalline counterpart. But second, and perhaps more significantly, the specific heat depends linearly on $T$, between 0 and $1 \mathrm{~K}$. What is most puzzling is that this anomalous behaviour of the specific heat is shared by a wide variety of glassy substances. Similar universality is also exhibited in the thermal conductivity data, which has a $T^{2}$-dependence.

Guided by these experimental results, Phillips (1972) and independently, Anderson et al (1972) postulated the existence of TLS in glasses. The point is, the free energy of a glass is characterized by deep valleys with multiple minima. The system gets trapped in one of these from which it has difficulty to extricate itself and is therefore, 


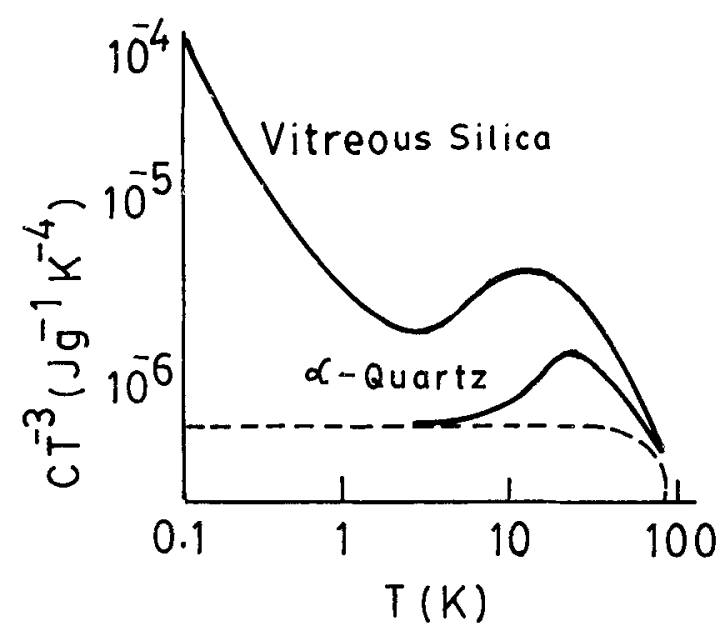

Figure 9. The heat capacity $C(T)$ of vitreous silica and crystalline quartz as a function of temperature $T$, plotted as $C / T^{3}$ against $T$.

found in a metastable state. However, it is conceivable that there could be two minima close to each other in the configuration space of the glass, and a group of atoms occupying one of these two adjacent minima can tunnel to the other, at very low temperatures $(0<T<1 \mathrm{~K})$. If $n\left(\Delta_{0}\right) \mathrm{d} \Delta_{0}$ represents the number of TLS with tunnel splittings between $\Delta_{0}$ and $\Delta_{0}+\mathrm{d} \Delta_{0}$, it is assumed that $n\left(\Delta_{0}\right)$ is a constant $n_{0}$. This simple assumption leads to the following expression for the specific heat:

$$
C(T)=n_{0} \int_{0}^{\infty}\left(\frac{\Delta_{0}^{2}}{4 K_{\mathrm{B}} T^{2}}\right) \operatorname{sech}^{2}\left(\frac{\Delta_{0}}{2 K_{\mathrm{B}} T}\right) \mathrm{d} \Delta_{0} .
$$

The integrand is simply the Schottky contribution (Phillips 1987) which, upon integration over $\Delta_{0}$, yields

$$
C(T)=\frac{1}{6} n_{0} \pi^{2} K_{\mathrm{B}}^{2} T .
$$

While the specific heat is a thermodynamic quantity, the thermal conductivity is a transport property, given by the relation

$$
\mathscr{K}=\frac{1}{3} C(T) V_{\mathrm{s}} l,
$$

where $V_{s}$ is the velocity of sound and $/$ the mean free path of phonons. The calculation is, however, somewhat more complicated and requires a 'golden rule' treatment for $l$, employing the coupling between TLS and the phonons (Phillips 1987). This coupling turns out to be the same as described in (7) and the analysis for $\mathscr{K}$ can therefore be carried out on the same line as in section 3. In fact, the theory outlined earlier is applicable to all the dynamical properties of insulating glasses at low temperatures. We may further note that like $\mathrm{H}$ in $\mathrm{Nb}(\mathrm{OH})_{x}$, the double-wells in glasses also have a pronounced asymmetry. 
We have discussed above in section 3 that the interaction between TLS and phonons is characterized by a spectral density $J(\omega)$ proportional to the $U$-term in (9). On the other hand, the term proportional to $K$ is also relevant if we deal with metallic glasses instead of insulating glasses (Black 1981). Thus, TLS in metallic glasses can be treated on the same footing as the method outlined earlier for nonadiabatic effects involving conduction electrons.

\section{Conclusions}

In summary, we have presented here strong evidence for quantum effects in the low-temperature diffusion of $\mathrm{H}$ in $\mathrm{Nb}(\mathrm{OH})_{x}$. These effects are unambiguously investigated when the dynamics of the system can be described by TLS. The coupling between the TLS and both electrons and phonons can be studied by a unified model based on what is known as the spin-boson Hamiltonian. The quantum effects lead to unusual temperature dependence of the diffusion constant. While one may think that $\mathrm{H}$ in $\mathrm{Nb}(\mathrm{OH})_{x}$ is a special tailor-made system, we have argued that the physical behaviour and the concomitant theoretical analysis are germane to generic two-level systems that are believed to characterize insulating and metallic glasses at low temperatures.

\section{Acknowledgement}

The review presented here is based on collaborative work done with Hermann Grabert, Herbert Schober and Tabish Qureshi.

\section{References}

Alefeld G and Volkl J (eds.) 1978 Hydrogen metals I (Berlin : Springer)

Anderson P W, Halperin B I and Verma C M 1972 Philos. Mag. 251

Black J L 1981 in Glassy metals I, Topics in Applied Physics (ed) H J Guntherodt and H Beck (Berlin: Springer) 46 p. 167

Dattagupta $S 1987$ in Relaxation phenomena in condensed matter physics (Ordando: Academic Press)

Dattagupta S, Grabert H and Jung R 1989 J. Phys. Cl 1405

Dattagupta S and Qureshi T 1991 Physica B174 262

Flynn C P and Stoneham A M 1970 Phys. Ret. BI 3966

Grabert H and Schober H R 1994 Hydrogen in metals II (ed) H Wipf (Heidelberg:Springer)

Grabert H and Wipf $\mathrm{H} 1990$ in Festkörper-proleme-Advances in solid state physics (ed) U Rossler (Braunschweig: Vieweg) $30 \mathrm{p} .1$

Hempelmann R, Richter D and Springer $T 1989$ IFF Bulletin of $K F A$ Jülich 35 p. 3

Holstein T 1959 Ann. Phys. (NY) 8325,343

Kagan Yu and Klinger M I 1974 J. Phys. C7 2791

Leggett A J. Chakravarty S, Fisher M P A, Dorsey A T. Garg A and Zwerger W 1987 Rev. Mod. Phys. 591

Margerl A, Rush J J, Rowe J M, Richter D and Wipf H 1983 Phys. Rev. B27 927

Morkel C. Wipf H and Neumaier K 1978 Phys. Rev. Lett. 40947

Narayanamurti V and Pohl R O 1970 Rev. Mod. Phys. 42201

Phillips W A 1972 J. Low Temp. Phys. 7351

Phillips W A 1987 Rep. Prog. Phys. 501657

Pohl R O, Love W F and Stephens R B 1974 Proc. 5 th int. conf. on amorphous and liquid semiconductors (ed) J Stuke and W Brenig (London: Taylor and Francis) p. 1121

Qureshi T 1994 Some models for quantum dissipatine systems. Ph.D. Thesis, Jawaharlal Nehru University, New Delhi 
Qureshi T and Dattagupta S 1994 Phys. Rev. B49 12848

Richter D 1983 Springer Tracts in Modern Physics (Heidelberg: Springer) Vol. 101

Springer T and Richter D 1987 Methods in experimental physics (New York: Academic Press) B23 p. 131

Steinbinder D, Wipf H, Magerl A, Richter D, Dianonux A I and Neumaier K 1988 Europhys. Lett. 6535

Wipf H and Neumaier K 1984 Phys. Rev. Lett. 521308

Wipf H, Steinbinder D, Neumaier K, Gutsmiedl P, Magerl A and Dianonux J 1987 Europhys. Lett. 41379

Zeller R C and Pohl R O 1971 Phys. Rev. B4 2029 